MATHEMATICS OF COMPUTATION

Volume 72, Number 244, Pages 1731-1746

S 0025-5718(03)01525-4

Article electronically published on May 14, 2003

\title{
CONVERGENCE OF THE GHOST FLUID METHOD FOR ELLIPTIC EQUATIONS WITH INTERFACES
}

\author{
XU-DONG LIU AND THOMAS C. SIDERIS
}

\begin{abstract}
This paper proves the convergence of the ghost fluid method for second order elliptic partial differential equations with interfacial jumps. A weak formulation of the problem is first presented, which then yields the existence and uniqueness of a solution to the problem by classical methods. It is shown that the application of the ghost fluid method by Fedkiw, Kang, and Liu to this problem can be obtained in a natural way through discretization of the weak formulation. An abstract framework is given for proving the convergence of finite difference methods derived from a weak problem, and as a consequence, the ghost fluid method is proved to be convergent.
\end{abstract}

\section{INTRODUCTION}

This paper proves the convergence of the ghost fluid finite difference method introduced in [13] for a second-order elliptic equation with discontinuous coefficients and given interfacial jumps, see also [5], [7]. The ghost fluid method (GFM) is simple, efficient and robust. One of the novelties, and advantages, of the method is the arm-by-arm splitting technique which makes the method in many dimensions as simple as in one dimension. Another advantage is that the resulting linear system of the method is the same as the linear system obtained from the simplest standard five point stencil finite difference method for the equation without discontinuous coefficients or given interfacial jumps. Therefore the resulting linear system is symmetric and positive definite, and can be efficiently solved. This finite difference method captures the sharp solution profile at the interfaces without smearing. This is a necessity in the development of effective schemes for problems involving interfaces. The reader interested in seeing numerical examples of the GFM may consult [13]. A variety of other approaches to problems with interfaces have been taken, 9, [10, 11, [14, 15, 16. An important application of the method here is for Hele-Shaw flow, see 8 .

The convergence proof starts with the formulation of the problem, in terms of a uniformly elliptic bilinear form. In the context of finite elements, a similar starting point has been taken in 12, 1], 3, 4. Existence and uniqueness of a weak solution $v$ follow immediately by elementary functional analysis. The solution space is the same as $H_{0}^{1}$, except for a different, but equivalent, inner product induced by the bilinear form. The source term includes in effect a delta distribution along

Received by the editor August 21, 2001 and, in revised form, May 3, 2002.

2000 Mathematics Subject Classification. Primary 65N12, 35J25.

Research partially supported by the National Science Foundation: DMS-9805546 (X.-D.L.) and DMS-9800888 (T.C.S.). 
the interface to account for the jump in the normal derivative. The solution of the interface problem is then recovered by the addition of a background function to correct for the jump in the solution across the interface as well as the boundary conditions. Discretizing this weak formulation in a natural way results in a discrete weak problem, which is equivalent to the finite difference method in 13 . As expected, the discrete weak formulation inherits the structural conditions (uniform boundedness, extension, consistency) in the discrete sense; hence existence, uniqueness, and uniform boundedness of the family $v^{h}$ of discrete solutions hold.

We then provide an abstract framework for the convergence proof. In addition to the uniform structural conditions for the weak problem and its discretizations, we postulate the existence of a uniformly bounded family of extension operators $T^{h}$ from the discrete spaces to the solution space, which satisfy a strong approximation property. This natural assumption implies that as the grid size $h$ goes to zero the image of the discrete space fills out the entire solution space. Finally, we impose weak consistency of the discrete problem with the original problem. Under these assumptions, the sequence of extended weak solutions $T^{h}\left(v^{h}\right)$ converges weakly to the solution $v$ in $H_{0}^{1}$. Guided by the abstract framework, we establish the convergence of the finite difference scheme of [13].

There is a similarity between the standard convergence proof of finite element methods and our approach. Both of them use structural conditions. For finite element methods, the structural conditions and weak consistency are inherited directly from the weak problem for the PDE, because the discrete bilinear forms are obtained by restriction to finite dimensional subspaces. Cea's lemma then says that the extended discrete solution is the closest function in the finite dimensional subspace to the true solution. This reduces convergence and error estimation to a problem in approximation theory. For finite difference methods, further approximations are made so that the discrete problem cannot be obtained by restriction to a finite dimensional subspace. In our case, the difference between the finite difference scheme and the finite element method is that point values of the coefficients are used rather than cell averages. Because the coefficients in our problem are discontinuous, the solution lies only in $H_{0}^{1}$ and not $H^{2} \cap H_{0}^{1}$, and therefore, we obtain convergence, but without a rate.

\section{EquATIONS AND WEAK FORMULATION}

Consider a bounded domain $\Omega \subset \mathbb{R}^{n}$ with smooth boundary, $\partial \Omega$. Let $\Gamma \subset \Omega$ be a smooth interface of codimension $n-1$, represented by the zero level-set of a smooth function $\phi(x)$, which is a signed distance function of the interface locally. We assume that $\phi$ divides $\Omega$ into disjoint subdomains, $\Omega^{-}=\{\phi<0\}$ and $\Omega^{+}=\{\phi>0\}$, with $\partial \Omega^{-}=\Gamma$. Thus, we may write $\Omega=\Omega^{+} \cup \Omega^{-} \cup \Gamma$. The unit normal vector of the interface is $n=\nabla \phi /|\nabla \phi|$, for $\phi(x)=0$, pointing from $\Omega^{-}$to $\Omega^{+}$.

We seek solutions of the variable coefficient Poisson equation away from the interface given by

$$
\nabla \cdot(\beta(x) \nabla u(x))=f(x), \quad x \in \Omega \backslash \Gamma,
$$

in which $x=\left(x_{1}, \ldots, x_{n}\right)$ denotes the spatial variables and $\nabla$ is the gradient operator. The coefficient $\beta(x)$ is assumed to be a positive definite, symmetric $n \times n$ matrix, the components of which are continuously differentiable on the closure of 
each disjoint subdomain, $\Omega^{-}$and $\Omega^{+}$, but they may be discontinuous across the interface $\Gamma$. It follows that there are positive constants $m<M$ with $m \mathbf{I} \leq \beta(x) \leq M \mathbf{I}$, where $\mathbf{I}$ stands for the $n \times n$ identity matrix. We suppose that on the interface, $\beta$ assumes the limiting values from within $\Omega^{-}$. The right-hand side $f(x)$ is assumed to lie in $L^{2}(\Omega)$.

Given functions $a$ and $b$ along the interface $\Gamma$, we prescribe the jump conditions

$$
\left\{\begin{array}{l}
{[u]_{\Gamma}(x) \equiv u^{+}(x)-u^{-}(x)=a(x),} \\
{\left[(\beta u)_{n}\right]_{\Gamma}(x) \equiv(\beta u)_{n}^{+}(x)-(\beta u)_{n}^{-}(x)=b(x),}
\end{array} \quad x \in \Gamma .\right.
$$

Note that $(\beta u)_{n}=n \cdot \beta \nabla u$, and the " \pm " superscripts refer to limits taken from within the subdomains $\Omega^{ \pm}$.

Finally, we prescribe boundary conditions

$$
u(x)=g(x), \quad x \in \partial \Omega,
$$

for a given function $g$ on the boundary.

We are going to use the usual Sobolev spaces $H_{0}^{1}(\Omega)$ and $H^{1}(\Omega)$, but instead of the usual inner product we choose one which is better suited to our problem:

$$
B[u, v]=\int_{\Omega} \beta \nabla u \cdot \nabla v .
$$

This induces a norm on $H_{0}^{1}(\Omega)$ which is equivalent to the usual one, thanks to the Poincaré inequality and the uniform bounds for the coefficient matrix.

Let $R_{\Gamma}$ and $R_{\partial \Omega}$ denote the restriction operators from $H^{1}(\Omega)$ to $L^{2}(\Gamma)$ and $L^{2}(\partial \Omega)$, respectively. Throughout this section, we shall always assume that our boundary data $a, b$ are the restrictions of functions $\widetilde{a}, \widetilde{b} \in H_{0}^{1}(\Omega)$, and that $g$ is the restriction of a function $\widetilde{g} \in H^{1}(\Omega)$ :

$$
a=R_{\Gamma}(\widetilde{a}), \quad b=R_{\Gamma}(\widetilde{b}), \quad \text { and } \quad g=R_{\partial \Omega}(\widetilde{g}) .
$$

To simplify the notation, from now on we will drop the tildes.

First let us consider the version of problem of (2.1a), (2.1b), (2.1c) with homogeneous boundary conditions for $u$ :

$$
\begin{cases}\nabla \cdot(\beta(x) \nabla u(x))=f(x), & x \in \Omega \backslash \Gamma, \\ {[u]_{\Gamma}(x)=0,} & x \in \Gamma, \\ {\left[(\beta u)_{n}\right]_{\Gamma}(x)=b(x),} & x \in \Gamma, \\ u(x)=0, & x \in \partial \Omega .\end{cases}
$$

Definition 2.1. A function $u \in H_{0}^{1}(\Omega)$ is a weak solution of (2.4) if it satisfies

$$
-B[u, \psi]=\int_{\Omega} f \psi+\int_{\Omega^{-}} \nabla \cdot(b \psi n),
$$

for all $\psi \in H_{0}^{1}(\Omega)$.

A classical solution of (2.4),$\left.u\right|_{\Omega^{ \pm}} \in C^{2}\left(\overline{\Omega^{ \pm}}\right)$, is easily seen to satisfy (2.5). The boundary condition $[u]_{\Gamma}=0$ is understood with the aid of $R_{\Gamma}$, and it is fulfilled since $u \in H_{0}^{1}(\Omega)$.

Theorem 2.1. Given $f \in L^{2}(\Omega)$ and $b \in H_{0}^{1}(\Omega)$, there exists a unique weak solution of (2.5) in $H_{0}^{1}(\Omega)$. 
Proof. The right-hand side of (2.5)

$$
F(\psi)=\int_{\Omega} f \psi+\int_{\Omega^{-}} \nabla \cdot(b \psi n)
$$

is a continuous linear functional on $H_{0}^{1}(\Omega)$. By the Riesz representation theorem, there exists a unique $u \in H_{0}^{1}(\Omega)$ such that $-B[u, \psi]=F(\psi)$, for all $\psi \in H_{0}^{1}(\Omega)$.

Next we reduce the general case (2.1a), (2.1b), (2.1c) to the homogeneous case. We will construct a unique solution of the problem in the class

$$
H(a, g)=\left\{u: u-g+a \chi_{\Omega^{-}} \in H_{0}^{1}(\Omega)\right\},
$$

in which $\chi_{\Omega^{-}}$is the characteristic function of $\Omega^{-}$. If $u \in H(a, g)$, then

$$
[u]_{\Gamma}=a \quad \text { and } \quad R_{\partial \Omega}(u)=g .
$$

Note that $H_{0}^{1}(\Omega)$ can be identified with $H(0,0)$, and so the following definition is consistent with the previous one.

Definition 2.2. A function $u \in H(a, g)$ is a weak solution of (2.1a), 2.1b), 2.1c) if $v=u-g+a \chi_{\Omega^{-}}$satisfies

$$
-B[v, \psi]=F(\psi)
$$

for all $\psi \in H_{0}^{1}(\Omega)$, where

$$
\begin{aligned}
F(\psi) & =F_{1}(\psi)+\cdots+F_{4}(\psi) \\
& =\int_{\Omega} f \psi+\int_{\Omega} \beta \nabla g \cdot \nabla \psi-\int_{\Omega^{-}} \beta \nabla a \cdot \nabla \psi+\int_{\Omega^{-}} \nabla \cdot(b \psi n) .
\end{aligned}
$$

A classical solution of (2.1a), 2.1b), 2.1c) is necessarily a weak solution.

Theorem 2.2. If $f \in L^{2}(\Omega), g \in H^{1}(\Omega)$, and $a, b \in H_{0}^{1}(\Omega)$, then there exists a unique weak solution of (2.6a), (2.6b), (2.1c) in $H(a, g)$.

Proof. The right-hand side of (2.6b) is a continuous linear functional on $H_{0}^{1}(\Omega)$. By the Riesz representation theorem, there exists a unique $v \in H_{0}^{1}(\Omega)$ such that $-B[v, \psi]=F(\psi)$, for all $\psi \in H_{0}^{1}(\Omega)$. Define the solution as $u=v+g-a \chi_{\Omega^{-}}$.

Remark 1 . We could replace $\int_{\Omega^{-}} \nabla \cdot(b \psi n)$ by $\int_{\Gamma} b \psi d s$ in (2.6b). Then the requirement for $b$ is reduced to $b \in L^{2}(\Gamma)$, which is a bit weaker than our assumption (2.3) for $b$. Our choice makes it easier to prove convergence of the finite difference scheme below.

\section{Abstract Form of Finite DifFEREnCE Methods}

In this section an abstract framework is given for proving convergence of finite difference methods for the elliptic interface problem introduced in the previous section.

For any $h>0$, let $H_{0}^{1, h}$ be a finite dimensional vector space with norm $\|\cdot\|_{h}$. This space should be thought of as a discrete approximation of the Sobolev space $H_{0}^{1}(\Omega)$ with grid size measured by the parameter $h$. 
On each finite dimensional space $H_{0}^{1, h}$, we suppose there exists a bounded extension operator $T^{h}: H_{0}^{1, h} \rightarrow H_{0}^{1}(\Omega)$ with the bound

$$
\left\|T^{h}\left(\psi^{h}\right)\right\|_{H_{0}^{1}(\Omega)} \leq C_{0}\left\|\psi^{h}\right\|_{h},
$$

for all $\psi^{h} \in H_{0}^{1, h}$, with $C_{0}$ independent of $h$.

Furthermore, we assume a strong approximation property. That is, given $\psi \in H_{0}^{1}(\Omega)$, there exists a sequence $\psi^{h} \in H_{0}^{1, h}$ such that

$$
T^{h}\left(\psi^{h}\right) \rightarrow \psi \quad \text { in } \quad H_{0}^{1}(\Omega), \quad \text { as } \quad h \rightarrow 0 .
$$

On each vector space $H_{0}^{1, h}$, we suppose there is a uniformly bounded family of bilinear forms $B^{h}[\cdot, \cdot]$ such that for every $u^{h}, v^{h} \in H_{0}^{1, h}$

$$
C_{1}\left\|u^{h}\right\|_{h}^{2} \leq B^{h}\left[u^{h}, u^{h}\right] \quad \text { and } \quad\left|B^{h}\left[u^{h}, v^{h}\right]\right| \leq C_{2}\left\|u^{h}\right\|_{h}\left\|v^{h}\right\|_{h},
$$

for positive constants $C_{1}, C_{2}$ independent of $h$. We also assume there exists a uniformly bounded family of linear functionals $F^{h}(\cdot)$ on $H_{0}^{1, h}$ such that for every $\psi^{h} \in H_{0}^{1, h}$

$$
\left|F^{h}\left(\psi^{h}\right)\right| \leq C_{3}\left\|\psi^{h}\right\|_{h},
$$

again with a constant $C_{3}$ independent of $h$.

Finally, we impose weak consistency with the weak problem (2.6a), 2.6b). For every pair of sequences $v^{h}, \psi^{h} \in H_{0}^{1, h}$ such that $T^{h}\left(v^{h}\right) \rightarrow v$ weakly in $H_{0}^{1}(\Omega)$ and $T^{h}\left(\psi^{h}\right) \rightarrow \psi$ strongly in $H_{0}^{1}(\Omega)$, we have that

$$
B^{h}\left[v^{h}, \psi^{h}\right] \rightarrow B[v, \psi]
$$

and

$$
F^{h}\left(\psi^{h}\right) \rightarrow F(\psi),
$$

where $B$ and $F$ are defined by (2.2) and (2.6b).

Under these general assumptions, we have the following:

Lemma 3.1. For every $h>0$, there exists a unique solution $v^{h} \in H_{0}^{1, h}$ of the discrete problem

$$
-B^{h}\left[v^{h}, \psi^{h}\right]=F^{h}\left(\psi^{h}\right),
$$

for every $\psi^{h} \in H_{0}^{1, h}$.

The sequence of extensions $T^{h}\left(v^{h}\right)$ of the family of discrete solutions converges weakly to $v=u-g+a \chi_{\Omega^{-}}$in $H_{0}^{1}(\Omega)$, where $u$ is the solution of the weak problem (2.6a), 2.6b).

Proof. The existence of a unique solution of the discrete problem (3.4) follows by the Lax-Milgram lemma.

By the estimates (3.2a), (3.2b), we obtain a uniform bound for the sequence of discrete solutions $v^{h}$ :

$$
\left\|v^{h}\right\|_{h} \leq C,
$$

with $C$ independent of $h$. Thus, using (3.1a), we have the bound

$$
\left\|T^{h}\left(v^{h}\right)\right\|_{H_{0}^{1}(\Omega)} \leq C .
$$

By weak compactness in the Hilbert space $H_{0}^{1}(\Omega)$, there is a subsequence $T^{h^{\prime}}\left(v^{h^{\prime}}\right)$ converging weakly to some $v \in H_{0}^{1}(\Omega)$. 
Now let $\psi \in H_{0}^{1}(\Omega)$ be given. Using the approximation property (3.1b), choose a sequence $\psi^{h} \in H_{0}^{1, h}$ such that

$$
T^{h}\left(\psi^{h}\right) \rightarrow \psi \quad \text { in } \quad H_{0}^{1}(\Omega) .
$$

By weak consistency (3.3a), 3.3b we find that the limit function $v$ satisfies (2.6a), (2.6b) . Finally, since this problem has a unique solution, it follows that the full sequence $T^{h}\left(v^{h}\right)$ converges weakly to $v$ in $H_{0}^{1}(\Omega)$.

Remark 2. The simplest way in which to obtain a consistent, and therefore convergent, scheme is to define

$$
B^{h}\left[v^{h}, \psi^{h}\right]=B\left[T^{h}\left(v^{h}\right), T^{h}\left(\psi^{h}\right)\right] \quad \text { and } \quad F^{h}\left(\psi^{h}\right)=F\left(T^{h}\left(\psi^{h}\right)\right) .
$$

This is essentially the method of finite elements, which then hinges on the choice of the extension operator $T^{h},[2]$.

However, the scheme under consideration in the next sections is not of this type, insofar as it originates from different approximations for $B$ and $F$.

\section{NumericAL METHOD}

In this section, we rederive the finite difference scheme from 13 for (2.1a), 2.1b), (2.1c), by discretizing the weak formulation (2.6a), 2.6b).

For the remainder of the paper we assume that the coefficient matrix is of the form $\beta \mathbf{I}$, for some scalar function. We also assume that the data functions $a$, $b, g, f$, all lie in $C^{1}(\bar{\Omega})$, with $a, b$ vanishing on $\partial \Omega$. For simplicity, we restrict ourselves to the special case of a rectangular domain $\Omega=\left(x_{W}, x_{E}\right) \times\left(y_{S}, y_{N}\right)$ in the plane. Given positive integers $I$ and $J$, set $\Delta x=\left(x_{E}-x_{W}\right) /(I+1)$ and $\Delta y=$ $\left(y_{N}-y_{S}\right) /(J+1)$, and define a uniform grid $\Omega^{h}=\left\{\left(x_{i}, y_{j}\right)\right\}$, where $x_{i}=x_{W}+i \Delta x$ and $y_{j}=y_{S}+j \Delta y$ for $i=0,1, \cdots, I+1$ and $j=0,1, \cdots, J+1$. The grid size is defined as $h=\min (\Delta x, \Delta y)$. The ratio $\Delta x / \Delta y$ is fixed when the grid size $h$ goes to zero.

The set of grid functions will be denoted by

$$
H^{1, h}=\left\{w^{h}=\left(w_{i, j}\right): 0 \leq i \leq I+1,0 \leq j \leq J+1\right\} .
$$

The discrete solution space is defined as

$$
H_{0}^{1, h}=\left\{\psi^{h}=\left(\psi_{i, j}\right) \in H^{1, h}: \psi_{i, j}=0 \text { on the grid boundary }\right\} .
$$

To construct the bilinear form on $H_{0}^{1, h}$, we discretize the coefficient $\beta$ in two ways as follows:

$$
\beta_{i+1 / 2, j}^{1}=\beta\left(x_{i+1 / 2}, y_{j}\right), \quad \beta_{i, j+1 / 2}^{2}=\beta\left(x_{i}, y_{j+1 / 2}\right) .
$$

For $w^{h} \in H^{1, h}$, define the usual finite difference operators

$$
\begin{aligned}
\left(\nabla_{x} w\right)_{i+1 / 2, j} & =\left(w_{i+1, j}-w_{i, j}\right) / \Delta x \\
\left(\nabla_{y} w\right)_{i, j+1 / 2} & =\left(w_{i, j+1}-w_{i, j}\right) / \Delta y
\end{aligned}
$$


For $v^{h}, \psi^{h} \in H_{0}^{1, h}$, the bilinear form is then given by

$$
\begin{aligned}
B^{h}\left[v^{h}, \psi^{h}\right]= & \sum_{j=1}^{J} \sum_{i=0}^{I} \beta_{i+1 / 2, j}^{1}\left(\nabla_{x} v\right)_{i+1 / 2, j}\left(\nabla_{x} \psi\right)_{i+1 / 2, j} \Delta x \Delta y \\
& +\sum_{i=1}^{I} \sum_{j=0}^{J} \beta_{i, j+1 / 2}^{2}\left(\nabla_{y} w\right)_{i, j+1 / 2}\left(\nabla_{y} \psi\right)_{i, j+1 / 2} \Delta x \Delta y .
\end{aligned}
$$

As in the continuous case, we use this to define a norm on $H_{0}^{1, h}$ :

$$
\left\|\psi^{h}\right\|_{h}^{2}=B^{h}\left[\psi^{h}, \psi^{h}\right] .
$$

Our next task will be to discretize the linear functional $F$ in 2.6b. The data functions naturally give rise to grid functions $a^{h}, b^{h}$, etc., by restriction to the grid $\Omega^{h}$. The normal vector is discretized by

$$
n^{h}=\left(n^{(1), h}, n^{(2), h}\right)=\left(\phi_{x}^{h}, \phi_{y}^{h}\right) /\left|\nabla \phi^{h}\right|,
$$

where, for $i=1, \cdots, I, j=1, \cdots, J$,

$$
\begin{aligned}
& \left(\phi_{x}\right)_{i, j}=\left(\phi_{i+1, j}-\phi_{i-1, j}\right) /(2 \Delta x), \\
& \left(\phi_{y}\right)_{i, j}=\left(\phi_{i, j+1}-\phi_{i, j-1}\right) /(2 \Delta y) .
\end{aligned}
$$

Hence $n^{h}$ is defined on all interior grid points. It will not be used on $\partial \Omega$.

We can immediately define

$$
F_{1}^{h}\left(\psi^{h}\right)=\sum_{i=1}^{I} \sum_{j=1}^{J} f_{i, j} \psi_{i, j} \Delta x \Delta y
$$

and

$$
F_{2}^{h}\left(\psi^{h}\right)=B^{h}\left[g^{h}, \psi^{h}\right]
$$

The other two pieces require the localization of integrals to the subdomain $\Omega^{-}$. First, we discretize the characteristic function:

$$
\chi_{i, j}= \begin{cases}1, & \text { if } \quad \phi_{i, j} \leq 0 \\ 0, & \text { if } \quad \phi_{i, j}>0\end{cases}
$$

Define

$$
\begin{aligned}
\chi_{i+1 / 2, j}^{1} & =\left(\chi_{i, j}\left(1-\theta_{i+1 / 2, j}\right)+\chi_{i+1, j} \theta_{i+1 / 2, j}\right) \\
\theta_{i+1 / 2, j} & = \begin{cases}\left|\phi_{i+1, j}\right| /\left(\left|\phi_{i, j}\right|+\left|\phi_{i+1, j}\right|\right), & \text { if }\left|\phi_{i, j}\right|+\left|\phi_{i+1, j}\right|>0 \\
0 & \text { otherwise. }\end{cases}
\end{aligned}
$$

The factor $\chi_{i+1 / 2, j}^{1} \Delta x$ approximates the length of the portion of the arm from $\left(x_{i}, y_{j}\right)$ to $\left(x_{i+1}, y_{j}\right)$ within $\Omega^{-}$. And also, let

$$
\begin{aligned}
\chi_{i, j+1 / 2}^{2} & =\left(\chi_{i, j}\left(1-\theta_{i, j+1 / 2}\right)+\chi_{i, j+1} \theta_{i, j+1 / 2}\right) \\
\theta_{i, j+1 / 2} & = \begin{cases}\left|\phi_{i, j+1}\right| /\left(\left|\phi_{i, j}\right|+\left|\phi_{i, j+1}\right|\right), & \text { if }\left|\phi_{i, j}\right|+\left|\phi_{i, j+1}\right|>0 \\
0 & \text { otherwise. }\end{cases}
\end{aligned}
$$


Now we define the remaining two pieces of the linear functional $F$ :

$$
\begin{aligned}
F_{4}^{h}\left(\psi^{h}\right)= & \sum_{j=1}^{J} \sum_{i=0}^{I} \nabla_{x}\left(b n^{(1)} \psi\right)_{i+1 / 2, j} \chi_{i+1 / 2, j}^{1} \Delta x \Delta y \\
& +\sum_{i=1}^{I} \sum_{j=0}^{J} \nabla_{y}\left(b n^{(2)} \psi\right)_{i, j+1 / 2} \chi_{i, j+1 / 2}^{2} \Delta x \Delta y
\end{aligned}
$$

and

$$
\begin{aligned}
F_{3}^{h}\left(\psi^{h}\right)= & -\sum_{j=1}^{J} \sum_{i=0}^{I} \beta_{i+1 / 2, j}^{1}\left(\nabla_{x} a\right)_{i+1 / 2, j}\left(\nabla_{x} \psi\right)_{i+1 / 2, j} \chi_{i+1 / 2, j}^{1} \Delta x \Delta y \\
& -\sum_{i=1}^{I} \sum_{j=0}^{J} \beta_{i, j+1 / 2}^{2}\left(\nabla_{y} a\right)_{i, j+1 / 2}\left(\nabla_{y} \psi\right)_{i, j+1 / 2} \chi_{i, j+1 / 2}^{2} \Delta x \Delta y .
\end{aligned}
$$

Using (4.6a), .., (4.6g), define

$$
F^{h}=F_{1}^{h}+\cdots+F_{4}^{h} .
$$

With the definitions (4.3) and (4.7) the discrete problem is formulated as in (3.4).

Next we show that the discrete weak formulation is the same as the finite difference scheme introduced in 13 .

Make the substitution $v^{h}=u^{h}-g^{h}+a^{h} \chi^{h}$, using (4.6c), in (3.4) to write

$$
-B^{h}\left[u^{h}, \psi^{h}\right]+B^{h}\left[g^{h}, \psi^{h}\right]-B^{h}\left[a^{h} \chi^{h}, \psi^{h}\right]=F_{1}^{h}\left(\psi^{h}\right)+\cdots+F_{4}^{h}\left(\psi^{h}\right) .
$$

We note right away that the second term on the left cancels with $F_{2}^{h}\left(\psi^{h}\right)$ on the right. We are going to apply summation by parts to remove the difference operators from the test vector $\psi^{h}$. The idea is expressed by the one-dimensional formula

$$
-\sum_{i=0}^{I} \alpha_{i+1 / 2}\left(\nabla_{x} \psi\right)_{i+1 / 2}=\sum_{i=1}^{I}\left(\nabla_{x} \alpha\right)_{i} \psi_{i},
$$

provided that $\psi_{0}=\psi_{I+1}=0$. Here, $\left(\nabla_{x} \alpha\right)_{i}=\left(\alpha_{i+1 / 2}-\alpha_{i-1 / 2}\right) / \Delta x$.

Using (4.9), the first term in (4.8) can be rewritten as

$$
-B^{h}\left[u^{h}, \psi^{h}\right]=\sum_{i=1}^{I} \sum_{j=1}^{J}\left[\nabla_{x}\left(\beta^{1} \nabla_{x} u\right)_{i, j}+\nabla_{y}\left(\beta^{2} \nabla_{y} u\right)_{i, j}\right] \psi_{i, j} \Delta x \Delta y .
$$

In the same way, we have, using (4.6d), (4.6e),

$$
F_{4}^{h}\left(\psi^{h}\right)=-\sum_{i=1}^{I} \sum_{j=1}^{J} b_{i, j}\left[n_{i, j}^{(1)}\left(\nabla_{x} \chi^{1}\right)_{i, j}+n_{i, j}^{(2)}\left(\nabla_{y} \chi^{2}\right)_{i, j}\right] \psi_{i, j} \Delta x \Delta y .
$$

To treat the remaining term on the left-hand side of (4.8), we use the following product rule for the difference operator:

$$
\begin{aligned}
& \nabla_{x}(a \chi)_{i+1 / 2, j}=\left(\nabla_{x} a\right)_{i+1 / 2, j} \chi_{i+1 / 2, j}^{1}+a_{i+1 / 2, j}^{1}\left(\nabla_{x} \chi\right)_{i+1 / 2, j}, \\
& a_{i+1 / 2, j}^{1}=a_{i+1, j}\left(1-\theta_{i+1 / 2, j}\right)+a_{i, j} \theta_{i+1 / 2, j},
\end{aligned}
$$


in which $\chi_{i+1 / 2, j}^{1}$ and $\theta_{i+1 / 2, j}$ were defined in (4.6d). Similarly, we have, using (4.6e),

$$
\begin{aligned}
& \nabla_{y}(a \chi)_{i, j+1 / 2}=\nabla_{y} a_{i, j+1 / 2} \chi_{i, j+1 / 2}^{2}+a_{i, j+1 / 2}^{2} \nabla_{x} \chi_{i, j+1 / 2}, \\
& a_{i, j+1 / 2}^{2}=a_{i, j+1}\left(1-\theta_{i, j+1 / 2}\right)+a_{i, j} \theta_{i, j+1 / 2} .
\end{aligned}
$$

It follows from this and (4.9) that

$$
\begin{aligned}
B^{h}\left[a^{h} \chi^{h}, \psi^{h}\right]+F_{3}^{h}\left(\psi^{h}\right) & \\
= & \sum_{i=0}^{I} \sum_{j=1}^{J} \beta_{i+1 / 2, j}^{1} a_{i+1 / 2, j}^{1}\left(\nabla_{x} \chi\right)_{i+1 / 2, j}\left(\nabla_{x} \psi\right)_{i+1 / 2, j} \Delta x \Delta y \\
& +\sum_{i=1}^{I} \sum_{j=0}^{J} \beta_{i, j+1 / 2}^{2} a_{i, j+1 / 2}^{2}\left(\nabla_{y} \chi\right)_{i, j+1 / 2}\left(\nabla_{y} \psi\right)_{i, j+1 / 2} \Delta x \Delta y \\
= & -\sum_{i=1}^{I} \sum_{j=1}^{J}\left[\nabla_{x}\left(\beta^{1} a^{1} \nabla_{x} \chi\right)_{i, j}+\nabla_{y}\left(\beta^{2} a^{2} \nabla_{x} \chi\right)_{i, j}\right] \psi_{i, j} \Delta x \Delta y .
\end{aligned}
$$

Combining (4.8), (4.10a), (4.10b), (4.10c), we obtain

$$
\begin{aligned}
\sum_{i=1}^{I} \sum_{j=1}^{J} & {\left[\nabla_{x}\left(\beta^{1} \nabla_{x} u\right)_{i, j}+\nabla_{y}\left(\beta^{2} \nabla_{y} u\right)_{i, j}\right] \psi_{i, j} \Delta x \Delta y } \\
= & -\sum_{i=1}^{I} \sum_{j=1}^{J}\left[\nabla_{x}\left(\beta^{1} a^{1} \nabla_{x} \chi\right)_{i, j}+\nabla_{y}\left(\beta^{2} a^{2} \nabla_{x} \chi\right)_{i, j}\right] \psi_{i, j} \Delta x \Delta y \\
& -\sum_{i=1}^{I} \sum_{j=1}^{J} b_{i, j}\left[n_{i, j}^{(1)} \nabla_{x}\left(\chi^{1}\right)_{i, j}+n_{i, j}^{(2)} \nabla_{y}\left(\chi^{2}\right)_{i, j}\right] \psi_{i, j} \Delta x \Delta y \\
& +\sum_{i=1}^{I} \sum_{j=1}^{J} f_{i, j} \psi_{i, j} \Delta x \Delta y .
\end{aligned}
$$

Since this must hold for all test vectors $\psi^{h} \in H_{0}^{1, h}$, we have shown that the finite difference scheme

$$
\begin{aligned}
\nabla_{x}\left(\beta^{1} \nabla_{x} u\right)_{i, j}+\nabla_{y}\left(\beta^{2} \nabla_{y} u\right)_{i, j}=- & \nabla_{x}\left(\beta^{1} a^{1} \nabla_{x} \chi\right)_{i, j}-\nabla_{y}\left(\beta^{2} a^{2} \nabla_{x} \chi\right)_{i, j} \\
& -b_{i, j}\left[n_{i, j}^{(1)}\left(\nabla_{x} \chi^{1}\right)_{i, j}+n_{i, j}^{(2)}\left(\nabla_{y} \chi^{2}\right)_{i, j}\right]+f_{i, j}
\end{aligned}
$$

holds at all interior grid points. Note that this is the scheme that was found in [13, see equation (77) therein.

Remark 3 . Here the passage from the weak formulation via summation by parts to the finite difference scheme is analogous to what is often done with PDE's.

\section{Convergence}

In this section, we establish the converge of the scheme (3.4).

Theorem 5.1. Let $\Omega \subset \mathbb{R}^{2}$ be a rectangle. Assume that the data functions $a, b$, $g, f$ all lie in $C^{1}(\bar{\Omega})$, with $a, b$ vanishing on $\partial \Omega$. Suppose that the coefficients have the form $\beta \mathbf{I}$. Then there is a family of linear extensions $T^{h}: H_{0}^{1, h} \rightarrow H_{0}^{1}(\Omega)$ which 
together with the bilinear forms $B^{h}$ (4.3) and the linear functionals $F^{h}$ (4.7) satisfy the structural conditions (3.1a), (3.1b), (3.2a), (3.2b), (3.3a), (3.3b).

The sequence of extended approximate solutions $T^{h}\left(v^{h}\right)$ of the discrete weak problem (3.4), (4.3), (4.7) converge weakly to the weak solution of the PDE (2.6a), (2.6b) in $H_{0}^{1}(\Omega)$.

Proof. The second statement follows from the first by Lemma 3.1. The next three subsections are devoted to the verification of the structural conditions: uniform boundedness, extension and approximation, and finally consistency.

\subsection{Uniform boundedness.}

Lemma 5.1. The family of bilinear forms (4.3) satisfies the uniform bounds (3.2a).

Proof. Because of our choice of norm in (4.4), the lower bound in (3.2a) is immediate. The upper bound in (3.2a) follows easily from the Cauchy-Schwarz inequality.

Lemma 5.2. The family of linear functionals (4.7) satisfies the uniform bound (3.2b).

Proof. We treat the four pieces $F_{1}^{h}, \ldots, F_{4}^{h}$ in turn.

By the Cauchy-Schwarz inequality, we have

$$
\left|F_{1}^{h}\left(\psi^{h}\right)\right| \leq\left(\sum_{i, j}\left|f_{i, j}\right|^{2} \Delta x \Delta y\right)^{1 / 2}\left(\sum_{i, j}\left|\psi_{i, j}\right|^{2} \Delta x \Delta y\right)^{1 / 2} .
$$

The first factor is just a Riemann sum for the $L^{2}$-norm of $f$, and so it is bounded by $2\|f\|_{L^{2}(\Omega)}$, for $h$ small enough. The second factor is estimated using the discrete version of the Poincaré inequality,

$$
\left(\sum_{i, j}\left|\psi_{i, j}\right|^{2} \Delta x \Delta y\right)^{1 / 2} \leq C(\Omega)\left(\sum_{i=0}^{I} \sum_{j=1}^{J}\left|\left(\nabla_{x} \psi\right)_{i+1 / 2, j}\right|^{2} \Delta x \Delta y\right)^{1 / 2},
$$

which follows as in the continuous case using summation by parts. This last sum is then bounded by $m^{-1}\left\|\psi^{h}\right\|_{h}$ because of the uniform lower bound for the coefficients $\beta$.

From (4.61), we can use the discrete product formula to rewrite $F_{4}^{h}$ as

$$
\begin{aligned}
& F_{4}^{h}\left(\psi^{h}\right) \\
& =\sum_{i=0}^{I} \sum_{j=1}^{J}\left[\left(b n^{(1)}\right)_{i+1, j}\left(\nabla_{x} \psi\right)_{i+1 / 2, j}+\nabla_{x}\left(b n^{(1)}\right)_{i+1 / 2, j} \psi_{i, j}\right] \chi_{i+1 / 2, j}^{1} \Delta x \Delta y \\
& \quad+\sum_{i=1}^{I} \sum_{j=0}^{J}\left[\left(b n^{(2)}\right)_{i, j+1}\left(\nabla_{x} \psi\right)_{i, j+1 / 2}+\nabla_{x}\left(b n^{(2)}\right)_{i, j+1 / 2} \psi_{i, j}\right] \chi_{i, j+1 / 2}^{2} \Delta x \Delta y
\end{aligned}
$$

Since $b$ and $\left(n^{(1)}, n^{(2)}\right)$ are in $C^{1}$ and $\left(\chi^{1}, \chi^{2}\right)$ is bounded, using the Cauchy-Schwarz inequality again, we get the bound

$$
\left|F_{4}^{h}\left(\psi^{h}\right)\right| \leq C\|b n\|_{L^{2}(\Omega)}\left\|\psi^{h}\right\|_{h}+C\|\nabla \cdot(b n)\|_{L^{2}(\Omega)}\left(\sum_{i, j}\left|\psi_{i, j}\right|^{2} \Delta x \Delta y\right)^{1 / 2} .
$$


Applying (5.1) to the last sum above, we obtain the desired bound for $F_{4}^{h}$.

In the same way, we get

$$
\left|F_{2}^{h}\left(\psi^{h}\right)\right| \leq C\|\beta \nabla g\|_{L^{2}(\Omega)}\left\|\psi^{h}\right\|_{h} \quad \text { and } \quad\left|F_{3}^{h}\left(\psi^{h}\right)\right| \leq C\|\beta \nabla a\|_{L^{2}(\Omega)}\left\|\psi^{h}\right\|_{h} .
$$

5.2. Strong approximation. In this section we define a uniformly bounded family of extension operators $T^{h}$ using the basic approach from the theory of finite elements. Then we verify the strong approximation property.

To this end, for each $h$ consider a triangulation of the domain $\Omega$ containing all triangles with vertices

$$
\left\{\left(x_{i}, y_{j}\right),\left(x_{i+1}, y_{j}\right),\left(x_{i}, y_{j+1}\right)\right\} \quad \text { or } \quad\left\{\left(x_{i}, y_{j}\right),\left(x_{i-1}, y_{j}\right),\left(x_{i}, y_{j-1}\right)\right\}
$$

based on the grid $\Omega^{h}$, as shown in Figure 1. For any grid point $\left(x_{i}, y_{j}\right) \in \Omega^{h}$, let $\eta_{i, j}^{h} \in H^{1}(\Omega)$ be the continuous, piecewise linear function which is equal to 1 at the grid point $\left(x_{i}, y_{j}\right)$ and equal to 0 at all other grid points. Given $w^{h} \in H^{1, h}$, the extension operator is defined as

$$
T^{h}\left(w^{h}\right)=\sum_{i, j} w_{i, j} \eta_{i, j} .
$$

Then $T^{h}: H^{1, h} \rightarrow H^{1}(\Omega)$ and $T^{h}: H_{0}^{1, h} \rightarrow H_{0}^{1}(\Omega)$. Although it is not required by the abstract framework, the extensions $T^{h}$ are linear operators.

Since $T^{h}\left(w^{h}\right)$ is the unique continuous linear interpolant on each triangle $\left\{\left(x_{i}, y_{j}\right),\left(x_{i \pm 1}, y_{j}\right),\left(x_{i}, y_{j \pm 1}\right)\right\}$, we have explicitly

$$
T^{h}\left(w^{h}\right)(x, y)=w_{i, j}+\left(\nabla_{x} w\right)_{i \pm 1 / 2, j}\left(x-x_{i \pm 1}\right)+\left(\nabla_{y} w\right)_{i, j \pm 1 / 2}\left(y-y_{j \pm 1}\right) .
$$

Given the coefficient function $\beta$ on $\Omega$, we write, using the definition (4.2),

$$
\beta^{1, h}=\left(\beta_{i+1 / 2, j}^{1}\right), \quad \beta^{2, h}=\left(\beta_{i, j+1 / 2}^{2}\right) .
$$

In general, given a discrete function $\beta^{1, h}$ defined at the half grid points $\left(x_{i+1 / 2}, y_{j}\right)$, as above, we define the piecewise constant extension

$$
S^{1, h}\left(\beta^{1, h}\right)(x, y)=\beta_{i+1 / 2, j}^{1}
$$

on every triangular cell having the horizontal edge from $\left(x_{i}, y_{j}\right)$ to $\left(x_{i+1}, y_{j}\right)$, see Figure 2. Similarly, given a discrete function $\beta^{2, h}$ defined at the half grid points $\left(x_{i}, y_{j+1 / 2}\right)$, we define

$$
S^{2, h}\left(\beta^{2, h}\right)(x, y)=\beta_{i, j+1 / 2}^{2}
$$

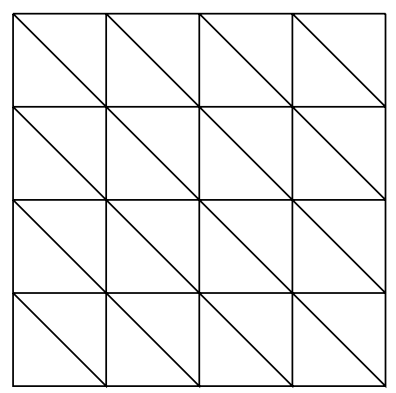

FIGURE 1. 

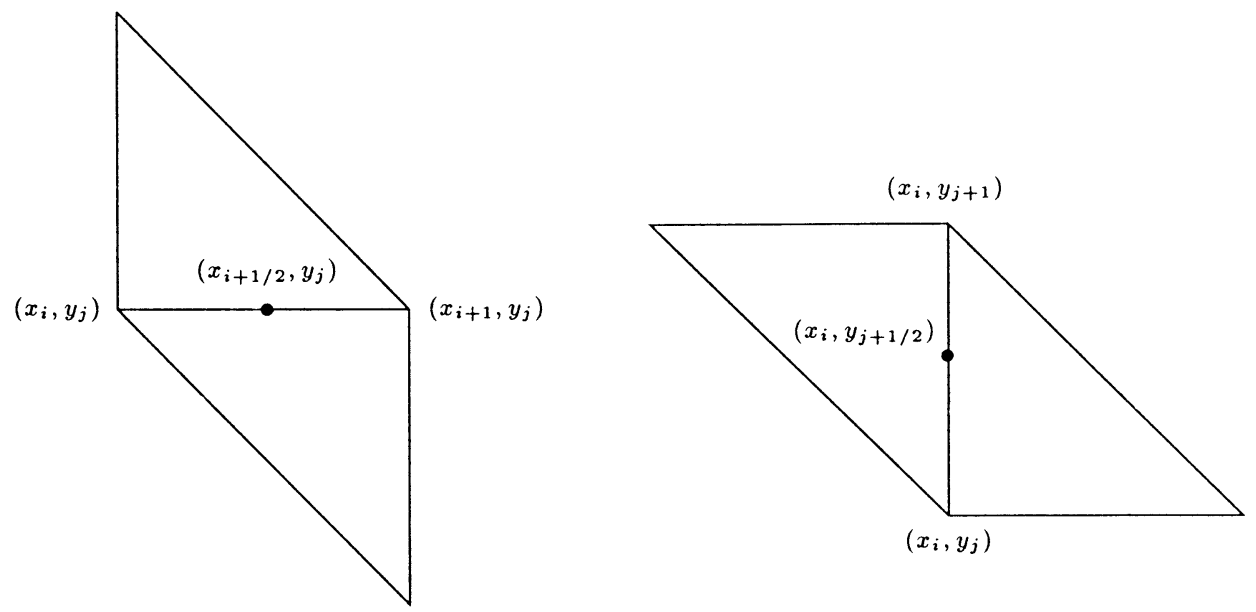

FIGURE 2.

on every triangular cell having the vertical edge from $\left(x_{i}, y_{j}\right)$ to $\left(x_{i}, y_{j+1}\right)$, see Figure 2.

The key to our estimates will be to replace sums by integrals. The following lemma summarizes the important formulas.

Lemma 5.3. With the definitions above, we have

$$
\begin{aligned}
& B^{h}\left[v^{h}, \psi^{h}\right]=\int_{\Omega} S^{1, h}\left(\beta^{1, h}\right) T^{h}\left(v^{h}\right)_{x} T^{h}\left(\psi^{h}\right)_{x} \\
&\left.+S^{2, h}\left(\beta^{2, h}\right) T^{h}\left(v^{h}\right)_{y} T^{h}\left(\psi^{h}\right)_{y}\right], \\
& F_{4}^{h}\left(\psi^{h}\right)=\int_{\Omega}\left[S^{1, h}\left(\chi^{1, h}\right) T^{h}\left(b^{h} n^{(1), h} \psi^{h}\right)_{x}\right. \\
&\left.+S^{2, h}\left(\chi^{2, h}\right) T^{h}\left(b^{h} n^{(2), h} \psi^{h}\right)_{y}\right], \\
& F_{3}^{h}\left(\psi^{h}\right)=-\int_{\Omega}\left[S^{1, h}\left(\beta^{1, h}\right) S^{1, h}\left(\chi^{1, h}\right) T^{h}\left(a^{h}\right)_{x} T^{h}\left(\psi^{h}\right)_{x}\right. \\
&\left.+S^{2, h}\left(\beta^{2, h}\right) S^{2, h}\left(\chi^{2, h}\right) T^{h}\left(a^{h}\right)_{y} T^{h}\left(\psi^{h}\right)_{y}\right] .
\end{aligned}
$$

Proof. Suppose that $\xi_{i+1 / 2, j}^{1}$ is defined for $i=0, \ldots, I, j=0, \ldots, J+1$, and $\xi_{i+1 / 2, j}^{1}=0$ for $j=0$ or $j=J+1$. Each point of the form $\left(x_{i+1 / 2}, y_{j}\right), i=1, \ldots, I$, $j=1, \ldots, J$, lies on the boundary of the two triangular cells sharing the arm from $\left(x_{i}, y_{j}\right)$ to $\left(x_{i+1}, y_{j}\right)$, and on these cells we have $\xi_{i+1 / 2, j}^{1}=S^{1, h}\left(\xi^{1, h}\right)(x, y)$. On the remaining cells, namely those with $j=0$ or $j=J+1$, we have $\xi_{i+1 / 2, j}^{1}=$ $S^{1, h}\left(\xi^{1, h}\right)=0$. Therefore, since the area of each pair of cells (where $S^{1, h}\left(\xi^{1, h}\right)$ could be nonzero) is equal to $\Delta x \Delta y$, we have

$$
\sum_{i=0}^{I} \sum_{j=1}^{J} \xi_{i+1 / 2, j}^{1} \Delta x \Delta y=\int_{\Omega} S^{1, h}\left(\xi^{1, h}\right) .
$$


Similarly, we have

$$
\sum_{i=1}^{I} \sum_{j=0}^{J} \xi_{i, j+1 / 2}^{2} \Delta x \Delta y=\int_{\Omega} S^{2, h}\left(\xi^{1, h}\right),
$$

provided that $\xi_{i, j+1 / 2}^{2}=0$ for $i=0$ or $i=I+1$.

Notice that the piecewise continuous extensions $S^{\alpha, h}$ are multiplicative in the sense that, for $\alpha=1,2, S^{\alpha, h}\left(a^{\alpha, h} b^{\alpha, h}\right)=S^{\alpha, h}\left(a^{\alpha, h}\right) S^{\alpha, h}\left(b^{\alpha, h}\right)$, for arbitrary $a^{\alpha, h}$, $b^{\alpha, h}$ defined at the half grid points. So using the formulas just derived, we have from (4.3) that

$$
\begin{aligned}
B^{h}\left[v^{h}, \psi^{h}\right] & =\int_{\Omega}\left[S^{1, h}\left(\beta^{1, h} \nabla_{x} v^{h} \nabla_{x} \psi^{h}\right)+S^{2, h}\left(\beta^{2, h} \nabla_{y} v^{h} \nabla_{y} \psi^{h}\right)\right] \\
= & \int_{\Omega}\left[S^{1, h}\left(\beta^{1, h}\right) S^{1, h}\left(\nabla_{x} v^{h}\right) S^{1, h}\left(\nabla_{x} \psi^{h}\right)\right. \\
& \left.\quad+S^{2, h}\left(\beta^{2, h}\right) S^{2, h}\left(\nabla_{y} v^{h}\right) S^{2, h}\left(\nabla_{y} \psi^{h}\right)\right] .
\end{aligned}
$$

By (5.2), we see that $S^{1, h}\left(\nabla_{x} \psi^{h}\right)=T^{h}\left(\psi^{h}\right)_{x}$ and $S^{2, h}\left(\nabla_{y} \psi^{h}\right)=T^{h}\left(\psi^{h}\right)_{y}$, and therefore, we have verified (5.3).

The proofs of the other two formulas are similar and will be omitted.

We are now ready to study the extensions.

Lemma 5.4. The family of extensions $T^{h}: H_{0}^{1, h} \rightarrow H_{0}^{1}(\Omega)$ satisfies the uniform estimates

$$
\frac{m}{M}\left\|\psi^{h}\right\|_{h}^{2} \leq\left\|T^{h} \psi^{h}\right\|_{H_{0}^{1}(\Omega)}^{2} \leq \frac{M}{m}\left\|\psi^{h}\right\|_{h}^{2}
$$

Proof. Let $\psi^{h} \in H_{0}^{1, h}$. By definition, we have

$$
\left\|T^{h} \psi^{h}\right\|_{H_{0}^{1}(\Omega)}^{2}=B\left[T^{h} \psi^{h}, T^{h} \psi^{h}\right] \quad \text { and } \quad\left\|\psi^{h}\right\|_{h}^{2}=B^{h}\left[\psi^{h}, \psi^{h}\right] .
$$

Moreover, by (5.3), we have

$$
B^{h}\left[\psi^{h}, \psi^{h}\right]=\int_{\Omega}\left[S^{1, h}\left(\beta^{1, h}\right) T^{h}\left(\psi^{h}\right)_{x}^{2}+S^{2, h}\left(\beta^{2, h}\right) T^{h}\left(\psi^{h}\right)_{y}^{2}\right] .
$$

Now by the uniform bounds on $\beta$, we have

$$
\beta(x, y) \leq \frac{M}{m} \beta\left(x^{\prime}, y^{\prime}\right),
$$

for arbitrary $(x, y),\left(x^{\prime}, y^{\prime}\right) \in \Omega$, and so

$$
\frac{m}{M} \beta \leq S^{1, h}\left(\beta^{1, h}\right), S^{2, h}\left(\beta^{2, h}\right) \leq \frac{M}{m} \beta .
$$

By (5.6), it follows that

$$
\frac{m}{M} B^{h}\left[\psi^{h}, \psi^{h}\right] \leq B\left[T^{h} \psi^{h}, T^{h} \psi^{h}\right] \leq \frac{M}{m} B^{h}\left[\psi^{h}, \psi^{h}\right] .
$$

Lemma 5.5. The extension operator $T^{h}: H_{0}^{1, h} \rightarrow H_{0}^{1}(\Omega)$ has the strong approximation property (3.1b). 
Proof. First let $\psi \in C_{0}^{1}(\bar{\Omega})$, and define $\psi^{h} \in H_{0}^{1, h}$ to be the grid function with values $\psi_{i, j}=\psi\left(x_{i}, y_{j}\right)$. Since $\psi \in C^{1}(\bar{\Omega}), \psi$ and its first derivatives are uniformly continuous on $\bar{\Omega}$. It follows from (5.2) that the piecewise constant functions $T^{h}\left(\psi^{h}\right)_{x}$ and $T^{h}\left(\psi^{h}\right)_{y}$ converge uniformly to $\psi_{x}$ and $\psi_{y}$, respectively, therefore also in $L^{2}(\omega)$ :

$$
\left\|\nabla\left(T^{h} \psi^{h}-\psi\right)\right\|_{L^{2}(\Omega)} \rightarrow 0,
$$

as $h \rightarrow 0$. Since $\beta$ has a uniform lower bound, this implies that

$$
\left\|T^{h} \psi^{h}-\psi\right\|_{H_{0}^{1}(\Omega)} \rightarrow 0
$$

as $h \rightarrow 0$.

Finally, the result for general $\psi \in H_{0}^{1}(\Omega)$ follows by density.

\subsection{Weak consistency.}

Lemma 5.6. The bilinear form (4.3) satisfies the weak consistency hypothesis (3.3a).

Proof. Suppose that $v^{h}, \psi^{h} \in H_{0}^{1, h}$ are sequences such that $T^{h}\left(v^{h}\right) \rightarrow v$ weakly in $H_{0}^{1}(\Omega)$ and $T^{h}\left(\psi^{h}\right) \rightarrow \psi$ strongly in $H_{0}^{1}(\Omega)$. Thus, we have that $\nabla T^{h}\left(v^{h}\right) \rightarrow \nabla v$ weakly in $L^{2}(\Omega)$ and $\nabla T^{h}\left(\psi^{h}\right) \rightarrow \nabla \psi$ strongly in $L^{2}(\Omega)$.

Recalling the definitions from the previous subsection, we have that the functions $S^{\alpha, h}\left(\beta^{\alpha, h}\right)(\alpha=1,2)$ are both uniformly bounded and converge pointwise to $\beta$ in $\Omega \backslash \Gamma$. Writing

$$
S^{1, h}\left(\beta^{1, h}\right) T^{h}\left(\psi^{h}\right)_{x}-\beta \psi_{x}=\left[S^{1, h}\left(\beta^{1, h}\right)-\beta\right] \psi_{x}+S^{1, h}\left(\beta^{1, h}\right)\left[T^{h}\left(\psi^{h}\right)_{x}-\psi_{x}\right],
$$

it follows by the dominated convergence theorem that $S^{1, h}\left(\beta^{1, h}\right) T^{h}\left(\psi^{h}\right)_{x} \rightarrow \beta \psi_{x}$ strongly in $L^{2}(\Omega)$.

Now, using Lemma (5.3), we have

$$
\begin{aligned}
B^{h}\left[v^{h}, \psi^{h}\right]= & \int_{\Omega}\left[S^{1, h}\left(\beta^{1, h}\right) T^{h}\left(v^{h}\right)_{x} T^{h}\left(\psi^{h}\right)_{x}\right. \\
& \left.\quad+S^{2, h}\left(\beta^{2, h}\right) T^{h}\left(v^{h}\right)_{y} T^{h}\left(\psi^{h}\right)_{y}\right] \\
= & \left\langle T^{h}\left(v^{h}\right)_{x}, S^{1, h}\left(\beta^{1, h}\right) T^{h}\left(\psi^{h}\right)_{x}\right\rangle_{L^{2}(\Omega)} \\
& \quad+\left\langle T^{h}\left(v^{h}\right)_{y}, S^{2, h}\left(\beta^{2, h}\right) T^{h}\left(\psi^{h}\right)_{y}\right\rangle_{L^{2}(\Omega)} \\
& \rightarrow\left\langle v_{x}, \beta \psi_{x}\right\rangle_{L^{2}(\Omega)}+\left\langle v_{y}, \beta \psi_{y}\right\rangle_{L^{2}(\Omega)} \\
= & B[v, \psi],
\end{aligned}
$$

as $h \rightarrow 0$.

Lemma 5.7. The linear functional $F$ defined in (4.7) satisfies the weak consistency hypothesis (3.3b).

Proof. Let $\psi \in H_{0}^{1}(\Omega)$ and suppose that $\psi^{h} \in H_{0}^{1, h}$ is a sequence such that $T^{h}\left(\psi^{h}\right) \rightarrow \psi$ strongly in $H_{0}^{1}(\Omega)$. We must show that $F^{h}\left(\psi^{h}\right) \rightarrow F(\psi)$.

To begin, we observe that it is enough to prove this for test functions in $C_{0}^{1}(\bar{\Omega})$. For any $\bar{\psi} \in C_{0}^{1}(\bar{\Omega})$, we can write

$$
\begin{aligned}
\left|F^{h}\left(\psi^{h}\right)-F(\psi)\right| & \leq\left|F^{h}\left(\psi^{h}-\bar{\psi}^{h}\right)\right|+\left|F^{h}\left(\bar{\psi}^{h}\right)-F(\bar{\psi})\right|+|F(\bar{\psi}-\psi)| \\
& \leq\left\|F^{h}\right\|\left\|\psi^{h}-\bar{\psi}^{h}\right\|_{h}+\left|F^{h}\left(\bar{\psi}^{h}\right)-F(\bar{\psi})\right|+\|F\|\|\bar{\psi}-\psi\|_{H_{0}^{1}(\Omega)} .
\end{aligned}
$$


By Lemma 5.4 we have

$$
\begin{aligned}
\left\|\psi^{h}-\bar{\psi}^{h}\right\|_{h} & \leq \frac{M}{m}\left\|T^{h}\left(\psi^{h}-\bar{\psi}^{h}\right)\right\|_{H_{0}^{1}(\Omega)} \\
& \leq \frac{M}{m}\left[\left\|T^{h}\left(\psi^{h}\right)-\psi\right\|_{H_{0}^{1}(\Omega)}+\left\|T^{h}\left(\bar{\psi}^{h}\right)-\bar{\psi}\right\|_{H_{0}^{1}(\Omega)}+\|\bar{\psi}-\psi\|_{H_{0}^{1}(\Omega)}\right] .
\end{aligned}
$$

By Lemma 5.2, the norms $\left\|F^{h}\right\|,\|F\|$ are uniformly bounded. Therefore, we have shown that

$$
\begin{aligned}
\left|F^{h}\left(\psi^{h}\right)-F(\psi)\right| \leq\left|F^{h}\left(\bar{\psi}^{h}\right)-F(\bar{\psi})\right| & +C\left[\left\|T^{h}\left(\psi^{h}\right)-\psi\right\|_{H_{0}^{1}(\Omega)}\right. \\
& \left.+\left\|T^{h}\left(\bar{\psi}^{h}\right)-\bar{\psi}\right\|_{H_{0}^{1}(\Omega)}+\|\bar{\psi}-\psi\|_{H_{0}^{1}(\Omega)}\right] .
\end{aligned}
$$

We claim that this can be made arbitrarily small for all $h<h_{0}$. By assumption, we have that $T^{h}\left(\psi^{h}\right)-\psi \rightarrow 0$ in $H_{0}^{1}(\Omega)$. By density, $\bar{\psi}$ can be chosen arbitrarily close to $\psi$ in $H_{0}^{1}(\Omega)$. By construction, we have that $T^{h}\left(\bar{\psi}^{h}\right)-\bar{\psi} \rightarrow 0$ in $H_{0}^{1}(\Omega)$. This covers all but the first term above. The first term can also be made small if the consistency condition is valid for $\bar{\psi} \in C_{0}^{1}(\bar{\Omega})$.

We now proceed to verify the consistency of $F$ under the assumption that $\psi \in$ $C_{0}^{1}(\bar{\Omega})$ by considering each individual piece.

By (4.6a) and the fact that $\psi \in C_{0}^{1}(\bar{\Omega})$, we see that $F_{1}^{h}\left(\psi^{h}\right)$ is simply a Riemann sum for $F_{1}(\psi)$, and thus $F_{1}^{h}\left(\psi^{h}\right) \rightarrow F_{1}(\psi)$, as $h \rightarrow 0$.

Given $g \in C^{1}(\bar{\Omega})$, we have that $T^{h}\left(g^{h}\right) \rightarrow g$ in $H_{0}^{1}(\Omega)$, as in the proof of Lemma 5.5. Thus, we may apply the result of Lemma 5.6 to conclude that

$$
F_{2}\left(\psi^{h}\right)=B^{h}\left[g^{h}, \psi^{h}\right] \rightarrow B[g, \psi]=F_{4}(\psi) .
$$

According to (5.4), we have

$$
F_{4}^{h}\left(\psi^{h}\right)=\int_{\Omega}\left[S^{1, h}\left(\chi^{1, h}\right) T^{h}\left(b^{h} n^{(1), h} \psi^{h}\right)_{x}+S^{2, h}\left(\chi^{2, h}\right) T^{h}\left(b^{h} n^{(2), h} \psi^{h}\right)_{y}\right] .
$$

Since $b, n, \psi \in C^{1}(\bar{\Omega})$, we have as in the proof of Lemma 5.5 that $\nabla T^{h}\left(b^{h} n^{h} \psi^{h}\right) \rightarrow$ $\nabla b n \psi$ in $L^{2}$. Moreover, $S^{\alpha, h}\left(\chi^{\alpha, h}\right)$ is uniformly bounded and tends to $\chi_{\Omega^{-}}$pointwise in $\Omega \backslash \Gamma$. Thus, it follows that $F^{h}\left(\psi^{h}\right) \rightarrow F(\psi)$.

The consistency of $F_{3}^{h}$ follows from the formula (5.5), in the same way.

\section{ACKNOWLEDGMENTS}

We thank S. Osher and R. Fedkiw for stimulating discussions. In particular,

S. Osher gave us the insightful suggestion to try the weak formulation.

\section{REFERENCES}

1. Bramble, J. and King, J. A finite element method for interface problems in domains with smooth boundaries and interfaces, Adv. Comput. Math. vol. 6, pp. 109-138, (1996). MR 98e:65094

2. Ciarlet, P.G. The finite element method for elliptic problems. North-Holland, New York (1978). MR 58:25001

3. Chen, Z. and Zou, J. Finite element methods and their convergence for elliptic and parabolic interface problems. Numer. Math. vol. 79, pp. 175-202 (1998). MR 99d:65313

4. Ewing, R. Li, Z., Lin, T. and Lin, Y. The immersed finite volume element methods for the elliptic interface problems. Math. Comput. Simulation vol. 50 pp. $63-76$ (1999). MR 2000g:65118 
5. Fedkiw, R., Aslam, T., and Xu, Shaojie. The Ghost Fluid Method for Deflagration and Detonation Discontinuities. J. Comput. Phys. vol. 154, pp. 393-427 (1999). MR 2000e:76096

6. Fedkiw, R., Aslam, T., Merriman, B., and Osher, S. A Non-Oscillatory Eulerian Approach to Interfaces in Multimaterial Flows (The Ghost Fluid Method). J. Comput. Phys. vol. 152, pp. 457-492 (1999). MR 2000c:76061

7. Fedkiw, R. and Liu, X.-D. The Ghost Fluid Method for Viscous Flows, Progress in Numerical Solutions of Partial Differential Equations. Arcachon, France, edited by M. Hafez, July 1998.

8. Hou, T., Li, Z., Osher, S., Zhao, H. A Hybrid Method for Moving Interface Problems with Application to the Hele-Shaw Flow. J. Comput. Phys., vol. 134, pp. 236-252 (1997). MR 98d:76128

9. Johansen, H. and Colella, P. A Cartesian Grid Embedded Boundary Method for Poisson's Equation on Irregular Domains. J. Comput. Phys., vol. 147, pp. 60-85 (1998). MR 99m:65231

10. LeVeque, R.J. and $\mathrm{Li}, \mathrm{Z}$. The Immersed Interface Method for Elliptic Equations with Discontinuous Coefficients and Singular Sources. SIAM J. Numer. Anal. vol. 31, pp. 1019-1044 (1994). MR 95g:65139

11. Li, Z. A Fast Iterative Algorithm for Elliptic Interface Problems. SIAM J. Numer. Anal., vol. 35, no. 1, pp. 230-254, (1998). MR 99b:65126

12. Li, Z., Lin, T., and $\mathrm{Wu}, \mathrm{X}$. New Cartesian grid methods for interface problems using finite element formulation. Preprint.

13. Liu, X.-D., Fedkiw, R., and Kang, M. A Boundary Condition Capturing Method for Poisson's Equation on Irregular Domains. J. Comput. Phys. vol. 160, pp. 151-178 (2000). MR 2001a:65152

14. Mulder, W., Osher, S., and Sethian, J.A. Computing Interface Motion in Compressible Gas Dynamics. J. Comput. Phys. vol. 100, pp. 209-228 (1992). MR 93a:76077

15. Peskin, C. Numerical Analysis of Blood Flow in the Heart. J. Comput. Phys., vol. 25, pp. 220-252 (1977). MR 58:9389

16. Peskin, C. and Printz, B. Improved Volume Conservation in the Computation of Flows with Immersed Elastic Boundaries., J. Comput. Phys., vol. 105, pp. 33-46 (1993). MR 93k:76081

Department of Mathematics, University of California, Santa Barbara, California 93106

E-mail address: xliu@math.ucsb.edu

Department of Mathematics, University of California, Santa Barbara, California 93106

E-mail address: sideris@math.ucsb.edu 